\title{
BMJ Open Factors associated with treatment and control of hypertension among elderly adults in Shenzhen, China: a large-scale cross-sectional study
}

Wenqing Ni, Xueli Yuan, Jia Zhang, Ping Li, Hong Min Zhang, Yan Zhang, Jian Xu

To cite: Ni W, Yuan X, Zhang J, et al. Factors associated with treatment and control of hypertension among elderly adults in Shenzhen, China: a large-scale crosssectional study. BMJ Open 2021;11:e044892. doi:10.1136/ bmjopen-2020-044892

- Prepublication history for this paper is available online. To view these files, please visit the journal online (http://dx.doi. org/10.1136/bmjopen-2020044892).

Received 17 September 2020 Accepted 28 July 2021

Check for updates

(C) Author(s) (or their employer(s)) 2021. Re-use permitted under CC BY-NC. No commercial re-use. See rights and permissions. Published by BMJ.

Department of Elderly Health Management, Shenzhen Center for Chronic Disease Control, Shenzhen, Guangdong, China

Correspondence to

Dr Jian Xu;

anniexu73@126.com

\section{ABSTRACT}

Objective Hypertension has become the leading cause of death worldwide. Data on hypertension management among Shenzhen elderly are sparse. Our study aims to investigate treated and controlled hypertension in Shenzhen elderly, and identify relevant predictors.

Design A cross-sectional study.

Setting Communities in Shenzhen, Guangdong, China. Participants A cross-sectional study was conducted. We employed a convenience sampling method to select participants; 124007 participants aged 65 years and older were recruited from January to December 2018 at local community health centres in Shenzhen.

Main outcome measures Data on treatment, control and influencing factors of hypertension were obtained from a standard questionnaire, physical measurements and biochemical analyses.

Results Prevalence of hypertension was 55.8\% among the sample population. Among this group of hypertensive patients, those undergoing hypertension treatment and those with hypertension under control were $54.4 \%$ and $32.3 \%$, respectively. Employing multivariate analysis, significant associations were found between treatment and older age, junior high school education and above $(\mathrm{OR}=1.25, \mathrm{p}<0.05)$, being widowed rather than being married or cohabiting $(0 R=1.28, p<0.05)$, engaging in physical activity $(0 R=1.14, p<0.05)$, ex-smoker $(\mathrm{OR}=1.19, \mathrm{p}<0.05)$, habitual drinker $(\mathrm{OR}=0.72$, $p<0.05$ ), history of cardiovascular disease (CVD) $(\mathrm{OR}=2.20, p<0.05)$ and comorbidities, with a higher probability for those with obesity $(0 R=1.89, p<0.05)$, central obesity $(O R=1.10, p<0.05)$, diabetes $(0 R=1.49$, $p<0.05)$ or dyslipidaemia $(0 R=1.20, p<0.05)$. Male sex $(\mathrm{OR}=0.91, \mathrm{p}<0.05)$, junior high school education and above $(0 R=1.28, p<0.05)$, engaging in physical activity $(\mathrm{OR}=1.06, p<0.05)$, history of $\mathrm{CVD}(\mathrm{OR}=1.82, \mathrm{p}<0.05)$ and individuals who had diabetes $(O R=1.52, p<0.05)$ or dyslipidaemia $(0 R=1.05, p<0.05)$ were associated with increased likelihood of control. Aged 80 years and older $(0 R=0.93, p<0.05)$, habitual drinker $(0 R=0.73, p<0.05)$ and central obesity $(\mathrm{OR}=0.94, p<0.05)$ were negatively associated with control of hypertension.

Conclusions We found a high prevalence of hypertension, but a low prevalence of treatment and control among Shenzhen elderly.
Strengths and limitations of this study

- This study is the first to evaluate the prevalence treatment and control of hypertension in a population sample aged 65 years and older from local communities in Shenzhen.

- Convenience sampling was used to enrol the population sample.

- No data were collected on diet or family history of hypertension, which may play a role in the treatment and control of hypertension.

\section{INTRODUCTION}

Hypertension is a global public health challenge and a major risk factor leading to stroke, myocardial infarction and heart failure. ${ }^{1}$ As is the case for many conditions, hypertension increases with age, with prevalence increasing from $27 \%$ in patients aged under 60 years to $74 \%$ in those aged over 80 years. ${ }^{2}$ The Framingham Heart Study ${ }^{3}$ showed that more than $90 \%$ of participants with normal blood pressure at age 55 years eventually develop hypertension in later years. By the year 2020, the projected number of people living in China aged 60 years or older will comprise $17.8 \%{ }^{4}$ With this rapidly ageing population, the prevalence of hypertension can only be expected to rise. Therefore, it is crucial to understand the current status of management of hypertension in elderly adults.

The management of hypertension in the elderly has many challenges, including agreement on threshold and target blood pressure levels, and the balancing of adverse effects and potential benefits of treatment. ${ }^{5}$ While extensive studies have been undertaken to identify risk factors for hypertension in predominantly middle-aged populations, there exist gaps in our understanding of the risk profiles and management of hypertension among the older population. A better 
understanding of the factors impacting treatment and control of hypertension in older adults is critical to the development of interventions to manage high blood pressure in this growing sector of the population. Therefore, the aim of our study was to investigate the treatment and control rate of hypertension among the elderly population in Shenzhen, China, and identify associated risk factors to provide evidence for disease prevention and control, and improve the quality of life of older hypertension patients. Strategies to target hypertension in the elderly population are additionally proposed.

\section{MATERIAL AND METHODS \\ Study population}

We used convenience sampling to select our population sample by recruiting people aged 65 years and older from the lists of all residents registered at local community health centres in Shenzhen, China, from January 2018 to December 2018. Recruitment activities include pasting posters or placing foldings in local community health centres and other public places. Electronic posters also be distributed via all the open WeChat groups of local community health centres' staff, to make the survey available to the close contacts easily. Moreover, the staff of the local community health centres recruited the elderly adults in their service community to participate in the survey by telephone. The eligibility criteria of participants were as follows: (1) having lived in Shenzhen for more than 6 months and (2) able to participate in the study and give informed consent. We excluded residents living in prisons. Initially, 141684 were recruited into the study, accounting for 36.9\% (141 684/383 700) of the resident population of elderly adults in Shenzhen based on the data from the 2015 population census. Data were collected in medical examination rooms at local community health centres in the participants'residential areas. We asked the participants to complete a questionnaire, provide a fasting blood sample and attend physical examinations. Participants excluded from the study, 17 677, were those who did not complete the questionnaire, provide a fasting blood sample or were unable to attend physical examinations. Finally, 124007 participants $(87.5 \%)$ were included in the final data analysis.

\section{Questionnaire survey}

Before the survey began, all investigators completed a training programme on the methods and process of the study. A manual of procedures was distributed, and detailed instructions for administration of the questionnaires, the taking of blood pressure and anthropometric measurements, and biological specimen collection and processing were provided.

Data were obtained during face-to-face interview in person 1 hour after blood collection. All participants completed a standardised questionnaire including sociodemographic status such as date of birth, sex, educational level and marital status; medical history such as history of previous diseases, operation history and history of trauma; family health history such as incidence of hypertension, diabetes, coronary heart disease, malignant tumour and stroke; lifestyle habits such as smoking status, amount of physical activity and alcohol consumption; and medication use under the supervision of trained general practitioners and nurses. Educational level was categorised into three groups according to the number of years of education: illiterate, no education; primary education, 1-6 years of education and junior high school education and above, 7 or more years of education.

In this study, we define the term "moderate to vigorous physical activity" to refer to at least some sweating and shortness of breath caused by engaging in physical activity, and the term 'light physical activity' to refer to no sweating or shortness of breath caused by engaging in physical activity. ${ }^{6}$ In addition, moderate to vigorous physical activity at least once a week was classified as 'yes' for physical activity status. For alcohol consumption status, participants reported themselves as habitual drinker (drinking at least once a day), non-habitual drinker (drinking six times a week to once a month) or non-drinker (almost never). ${ }^{7}$ For smoking status, we categorised participants as current smoker, ex-smoker and never-smoker, as described elsewhere. ${ }^{8}$

\section{Physical examination}

Anthropometric examinations were taken in the morning on participants who had fasted overnight, following which body measurements were taken by trained examiners based on a standardised protocol. Height and weight were measured using analogue scales with the participants wearing light clothing without footwear. Waist circumference (WC) was measured at the end of normal expiration at the midpoint level of the midaxillary line between the 12th rib head and the superior anterior iliac spine. Body mass index (BMI) was calculated by dividing body weight (in kilograms) by the square of height (in $\mathrm{m})$. Blood pressure were measured in both arms and recorded the higher one. Calibrated electronic sphygmomanometers were used to measure blood pressure on the arm supported at heart level with sitting position, carried out twice. The average of the two measurements was used for the statistical analysis. To obtain accurate readings, the participants were asked to rest for at least $5 \mathrm{~min}$ before the measurement, or, if having engaged in excessive exercise prior to the visit, for at least $30 \mathrm{~min}$ before the measurement. Untreated subjects with a blood pressure of at least 140/90 mm $\mathrm{Hg}$ were seen again at a second visit within 2 weeks, and if still $\geq 140$ and/or $90 \mathrm{~mm} \mathrm{Hg}$, they were seen a third time again within two further weeks. Those with high blood pressure at the first visit and who had normal blood pressure for both systolic blood pressure (SBP) $(<140 \mathrm{~mm} \mathrm{Hg})$ and diastolic blood pressure (DBP) $(<90 \mathrm{~mm} \mathrm{Hg})$ at the second or third visit were considered normotensive. 


\section{Blood sample collection and biochemical analyses}

Participant venous blood samples were taken after at least 8 hours of overnight fasting. All blood samples were analysed at the clinical laboratories of grade 2 hospitals to which the community health centres were directly affiliated. All the laboratories involved had successfully completed a standardisation and competency programme. Fasting venous blood was drawn from subjects for the measurements of levels of total cholesterol (TC), triglycerides (TG), low-density lipoprotein cholesterol (LDL-C) and high-density lipoprotein cholesterol (HDL-C) by automatic biochemistry analyser. TC and TG were estimated using enzymatic methods with commercially available reagents, while HDL and LDL were measured using a timed-endpoint colorimetric method. Fasting blood samples were biochemically analysed within a maximum of 4 hours after being drawn. Glucose oxidase measurements were used to ascertain the fasting blood glucose (FBG) level.

\section{Definitions}

A diagnosis of hypertension was considered when three consecutive high readings ( $\geq 140 \mathrm{SBP}$ and/or $\geq 90 \mathrm{~mm} \mathrm{Hg}$ DBP) with 2-week intervals were registered or treatment with antihypertensive medication within the previous 2 weeks was self-reported. ${ }^{9}$ Participants were considered to be undergoing treatment for hypertension if they answered 'yes' to the question 'Because of your high blood pressure/hypertension, are you now taking prescribed medicine?' Controlled hypertension was defined as SBP $<140 \mathrm{~mm} \mathrm{Hg}$ and DBP $<90 \mathrm{~mm} \mathrm{Hg}$, and reported use of antihypertensive medication during the survey period. ${ }^{910}$ Participants were regarded as diabetic if one of the following three conditions was met: (1) previous diagnosis by professional doctors; (2) $\mathrm{FBG} \geq 7.0 \mathrm{mmol} / \mathrm{L}$; or (3) 2-hour plasma glucose level $\geq 11.1 \mathrm{mmol} / \mathrm{L}$. ${ }^{11} \mathrm{TC}$, LDL-C, HDL-C and TG levels were classified on the basis of the 2016 Chinese Guideline for the Management of Dyslipidemia in Adults. ${ }^{12}$ It defines high TC as $\geq 6.22 \mathrm{mmol} / \mathrm{L}$, high LDL-C as $\geq 4.14 \mathrm{mmol} / \mathrm{L}$, low HDL-C as $<1.04 \mathrm{mmol} / \mathrm{L}$ and high TG as $\geq 2.26 \mathrm{mmol} / \mathrm{L}$. In the present study, we defined dyslipidaemia as the presence of one or more abnormal serum lipid concentrations or use of antidyslipidaemia medications in the previous 2 weeks.

Based on the criteria of weight for adults released by the Ministry of Health of China (WS/T 428-2013), individuals were categorised into four groups: $\mathrm{BMI}<18.5 \mathrm{~kg} /$ $\mathrm{m}^{2}$, low weight; $18.5 \mathrm{~kg} / \mathrm{m}^{2} \leq \mathrm{BMI}<24.0 \mathrm{~kg} / \mathrm{m}^{2}$, normal weight; $24.0 \mathrm{~kg} / \mathrm{m}^{2} \leq \mathrm{BMI}<28.0 \mathrm{~kg} / \mathrm{m}^{2}$, overweight and BMI $\geq 28.0 \mathrm{~kg} / \mathrm{m}^{2}$, obese. Men with WC $\geq 90 \mathrm{~cm}$ and women with $\mathrm{WC} \geq 85 \mathrm{~cm}$ were defined as having central obesity.

\section{Statistical analyses}

We collected descriptive statistics for all the variables, including continuous variables, expressed as means and $\mathrm{SD}$, and categorical variables, expressed as numbers and percentages. Categorical variables between groups were compared using a $\chi^{2}$ test. Multivariate logistic regression analysis was performed to explore the association between treatment and control of hypertension, and associated risk factors. In the multivariate logistic regression model, the treatment or control of hypertension was defined as the dependent variable, and age, sex, education level, marital status, smoking status, alcohol consumption, physical activity status, BMI, central obesity, diabetes, diagnosis of dyslipidaemia and history of cardiovascular disease (CVD) were defined as the independent variables. SAS software V.9.4 (SAS Institute) was used to perform all statistical analyses. Tests were two sided, and $\mathrm{p}<0.05$ was considered to be statistically significant.

\section{Participants and public involvement}

Neither the study participants nor the public were involved in the design, recruitment or conduct of the study. All the participants had the option of receiving a health check and biochemical results when they visited the local community health centres.

Written informed consent was received by all participants before the collection of data and conducting of the research. Where participants were illiterate, we obtained written informed consent from their proxies.

\section{RESULTS}

\section{Sociodemographic and other characteristics of participants}

Of the 124007 participants, $44.1 \% \quad(\mathrm{n}=54649)$ were male and $55.9 \%(\mathrm{n}=69358)$ were female, the mean age was $71.3 \pm 5.6,56.3 \%$ had attained a junior school education or above, $96.2 \%$ were married or cohabiting, $76.9 \%$ reported engaging in regular physical activities and $4.2 \%$ reported having the history of CVD (table 1). Current smokers accounted for $8.2 \%$, and habitual drinkers accounted for $6.4 \%$ (table 1). In terms of anthropometric measurements, the means of average BMI, SBP, DBP, WC, FBG, TC, TG, LDL-C and HDL-C levels for all 124007 participants were $23.8 \pm 3.2 \mathrm{~kg} / \mathrm{m}^{2}, 134.7 \pm 17.7 \mathrm{~mm}$ $\mathrm{Hg}, 77.2 \pm 10.3 \mathrm{~mm} \mathrm{Hg}, 85.1 \pm 8.8 \mathrm{~cm}, 6.0 \pm 1.9 \mathrm{mmol} / \mathrm{L}$, $5.2 \pm 2.1 \mathrm{mmol} / \mathrm{L}, 1.6 \pm 1.1 \mathrm{mmol} / \mathrm{L}, 3.1 \pm 1.1 \mathrm{mmol} / \mathrm{L}$ and $1.4 \pm 0.5 \mathrm{mmol} / \mathrm{L}$, respectively (table 1 ).

\section{Treatment and control rates in subgroups}

The prevalence of hypertension was $55.8 \%$. Of those individuals with hypertension, $54.4 \%$ were receiving treatment, and $32.3 \%$ had blood pressure under control. Table 2 lists the treatment and control of hypertension by sociodemographic grouping, lifestyle, clinical and anthropometric characteristics. The treatment rate demonstrated no difference between males and females. However, males had a significantly higher rate of control than females. Higher treatment and control rates were also found among those who had attained junior school education or above, engaged in regular physical activity, were ex-smokers and had history of CVD, or diabetes or dyslipidaemia. Participants who were single had the 
Table 1 Sociodemographic, anthropometric, lifestyle and clinical characteristics of older adults living in Shenzhen $(n=124007)$

\begin{tabular}{|c|c|c|c|}
\hline Characteristics & General $(\mathrm{N}=124007)$ & Characteristics & General $(\mathrm{N}=124007)$ \\
\hline Age (years) & $71.3 \pm 5.6$ & Physical activity, n (\%) & \\
\hline BMI $\left(\mathrm{kg} / \mathrm{m}^{2}\right)$ & $23.8 \pm 3.2$ & Yes & $95338(76.9)$ \\
\hline SBP $(\mathrm{mm} \mathrm{Hg})$ & $134.7 \pm 17.7$ & No & $28669(23.1)$ \\
\hline $\mathrm{DBP}(\mathrm{mm} \mathrm{Hg})$ & $77.2 \pm 10.3$ & History of cardiovascular disease, $\mathrm{n}(\%)$ & \\
\hline WC $(\mathrm{cm})$ & $85.1 \pm 8.8$ & Yes & $5192(4.2)$ \\
\hline FBG (mmol/L) & $6.0 \pm 1.9$ & No & $118815(95.8)$ \\
\hline $\mathrm{TC}(\mathrm{mmol} / \mathrm{L})$ & $5.2 \pm 2.1$ & Smoking status, n (\%) & \\
\hline $\mathrm{TG}(\mathrm{mmol} / \mathrm{L})$ & $1.6 \pm 1.1$ & Current smoker & $10163(8.2)$ \\
\hline LDL-C (mmol/L) & $3.1 \pm 1.1$ & Ex-smoker & $7662(6.2)$ \\
\hline HDL-C (mmol/L) & $1.4 \pm 0.5$ & Never-smoker & $106182(85.6)$ \\
\hline Sex, n (\%) & & Drinking habit, n (\%) & \\
\hline Male & $54649(44.1)$ & Non-drinker & $103388(83.4)$ \\
\hline Female & $69358(55.9)$ & Non-habitual drinker & $12737(10.2)$ \\
\hline Education level, n (\%) & & Habitual drinker & $7882(6.4)$ \\
\hline Illiterate & $10054(8.1)$ & & \\
\hline Primary education & $44096(35.6)$ & & \\
\hline Junior school education and above & $69857(56.3)$ & & \\
\hline \multicolumn{4}{|l|}{ Marital status, $\mathrm{n}(\%)$} \\
\hline Married or cohabiting & $119314(96.2)$ & & \\
\hline Widowed & $3623(2.9)$ & & \\
\hline Divorced & $565(0.5)$ & & \\
\hline Single & $505(0.4)$ & & \\
\hline
\end{tabular}

BMI, body mass index; DBP, diastolic blood pressure; FBG, fasting blood glucose; HDL-C, high-density lipoprotein cholesterol; LDL-C, low-density lipoprotein cholesterol; SBP, systolic blood pressure; TC, total cholesterol; TG, triglycerides; WC, waist circumference.

lowest treatment rates when compared with their nontreated counterparts. Participants aged 65-69 years had the lowest treatment rates compared with older participants. Habitual drinkers had the lowest treatment and control rates. Participants with obesity or central obesity had higher treatment rates and lower control rates.

\section{Results of multivariate analysis of factors associated with treatment and control of hypertension}

The results of multivariate logistic regression of hypertension treatment and control according to selected sociodemographic and other potential factors are presented in table 3. Participants with the highest educational attainment $(\mathrm{OR}=1.25,95 \%$ CI 1.18 to 1.32$)$ were more likely to be receiving treatment for hypertension compared with participants of low educational level. Being widowed $(\mathrm{OR}=1.28,95 \% \mathrm{CI}=1.17$ to 1.40$)$ was a protective factor for treatment of hypertension while being single ( $\mathrm{OR}=0.58,95 \% \mathrm{CI}=0.45$ to 0.74$)$ was negatively associated with hypertension treatment. Older age, engaging in physical activity $(\mathrm{OR}=1.14,95 \% \mathrm{CI}=1.10$ to 1.18$)$, being an ex-smoker $(\mathrm{OR}=1.19,95 \% \mathrm{CI}=1.11$ to 1.27$)$ and history of CVD (OR=2.20, 95\% CI=2.04 to 2.37) were associated with higher likelihood of receiving treatment for hypertension, while non-habitual drinker $(\mathrm{OR}=0.89,95 \% \mathrm{CI}=0.85$ to 0.94$)$ or habitual drinker $(\mathrm{OR}=0.72,95 \% \mathrm{CI}=0.68$ to 0.77$)$ was associated with lower likelihood of receiving treatment for hypertension. Compared with individuals without comorbidities, having comorbidities was also associated with higher rate of treatment: overweight $(\mathrm{OR}=1.64,95 \% \mathrm{CI}=1.48$ to 1.82$)$, obesity $(\mathrm{OR}=1.89,95 \% \mathrm{CI}=1.68$ to 2.11$)$, central obesity $(\mathrm{OR}=1.10,95 \% \mathrm{CI}=1.06$ to 1.14$)$, diabetes $(\mathrm{OR}=1.49$, $95 \% \mathrm{CI}=1.44$ to 1.54$)$ and dyslipidaemia (OR=1.20, 95\% $\mathrm{CI}=1.16$ to 1.24$)$. For those with hypertension, junior school education and above $(\mathrm{OR}=1.28,95 \% \mathrm{CI}=1.20$ to 1.36$)$, engaging in physical activity ( $\mathrm{OR}=1.06,95 \%$ $\mathrm{CI}=1.02$ to 1.10$)$ and history of CVD (OR=1.82, 95\% $\mathrm{CI}=1.71$ to 1.96$)$, diabetes $(\mathrm{OR}=1.52,95 \% \quad \mathrm{CI}=1.47$ to1.58) or dyslipidaemia ( $\mathrm{OR}=1.05,95 \% \mathrm{CI}=1.03$ to 1.09$)$ were positively associated with the control of hypertension. In contrast, female sex $(\mathrm{OR}=0.91,95 \% \mathrm{CI}=0.88$ to $0.95)$, aged 80 or above $(\mathrm{OR}=0.93,95 \% \mathrm{CI}=0.88$ to 0.98$)$, non-habitual drinker $(\mathrm{OR}=0.92,95 \% \mathrm{CI}=0.87$ to 0.97$)$, habitual drinker $(\mathrm{OR}=0.73,95 \% \mathrm{CI}=0.68$ to 0.79$)$ and central obesity $(\mathrm{OR}=0.94,95 \% \mathrm{CI}=0.91$ to 0.98$)$ were negatively associated with the control of hypertension. 
Table 2 Treatment and control of hypertension in older adults diagnosed with hypertension living in Shenzhen, according to sociodemographic, lifestyle, clinical and anthropometric characteristics $(n=69207)$

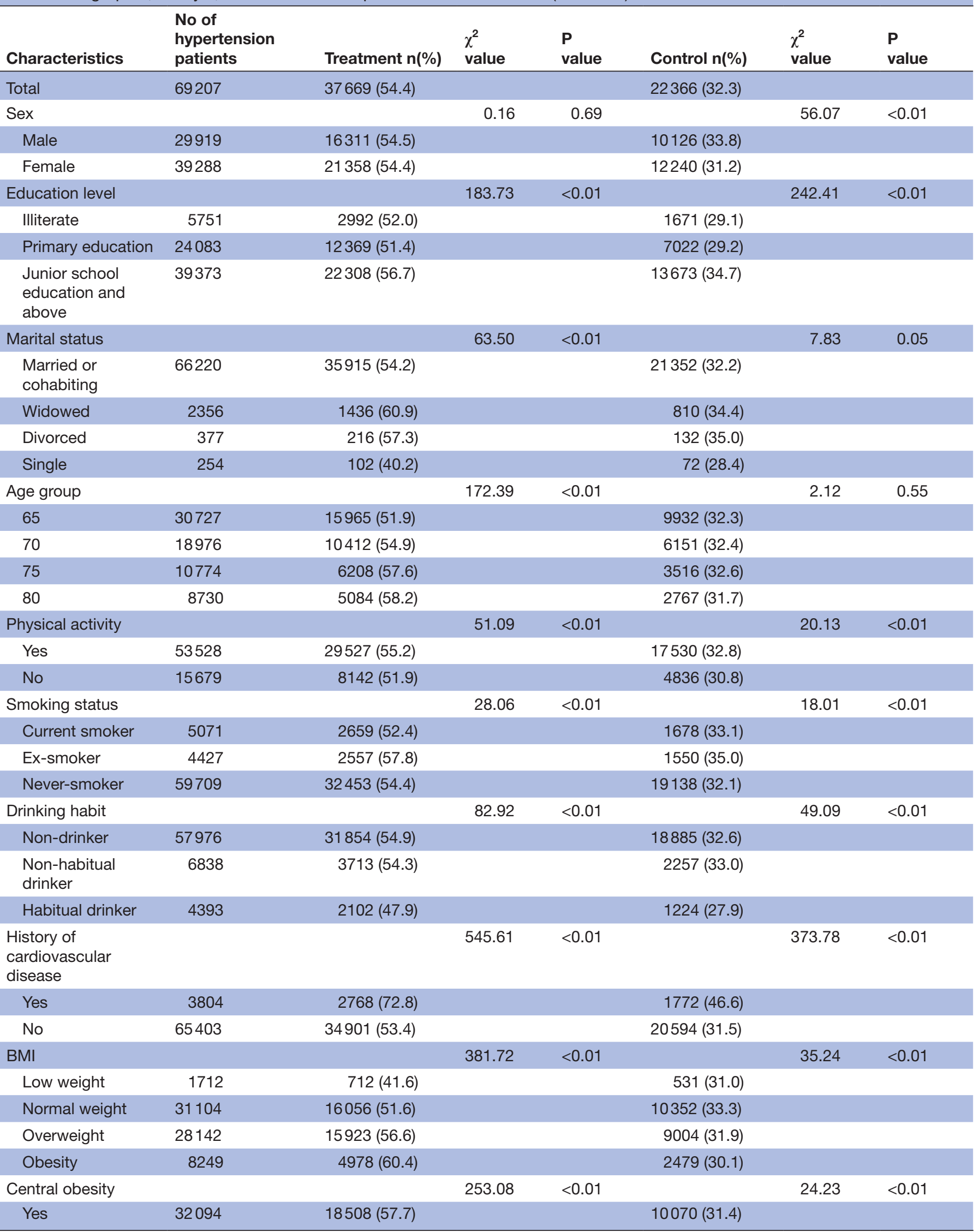

Continued 
Table 2 Continued

\begin{tabular}{|c|c|c|c|c|c|c|c|}
\hline Characteristics & $\begin{array}{l}\text { No of } \\
\text { hypertension } \\
\text { patients }\end{array}$ & Treatment n(\%) & $\begin{array}{l}\chi^{2} \\
\text { value }\end{array}$ & $\begin{array}{l}P \\
\text { value }\end{array}$ & Control n(\%) & $\begin{array}{l}\chi^{2} \\
\text { value }\end{array}$ & $\begin{array}{l}\mathbf{P} \\
\text { value }\end{array}$ \\
\hline No & 37113 & $19161(51.6)$ & & & $12296(33.1)$ & & \\
\hline Diabetes & & & 688.03 & $<0.01$ & & 603.08 & $<0.01$ \\
\hline Yes & 19263 & 12025 (62.4) & & & 7580 (39.4) & & \\
\hline No & 49944 & 25644 (51.4) & & & 14786 (29.6) & & \\
\hline Yes & 33416 & $19210(57.5)$ & & & 11138 (33.3) & & \\
\hline No & 35791 & 18459 (51.6) & & & 11228 (31.4) & & \\
\hline
\end{tabular}

Definition: A diagnosis of hypertension was considered when three consecutive high readings ( $\geq 140$ systolic and/or $\geq 90 \mathrm{~mm} \mathrm{Hg}$ diastolic) with 2-week intervals were registered or treatment with antihypertensive medication within the previous 2 weeks was self-reported. Participants were considered to be undergoing treatment if they answered 'yes' to the question 'Because of your high blood pressure/ hypertension, are you now taking prescribed medicine?' Controlled hypertension was defined as SBP $<140 \mathrm{~mm} \mathrm{Hg} \mathrm{and} \mathrm{DBP}<90 \mathrm{~mm}$ $\mathrm{Hg}$, and reported use of antihypertensive medication during the survey. $\mathrm{BMI}$, body mass index; DBP, diastolic blood pressure; SBP, systolic blood pressure.

\section{DISCUSSION}

Hypertension is the leading modifiable risk factor for CVD, which is the leading cause of death in China. ${ }^{13} 14$ The burden of hypertension and CVD on the healthcare system in China is increasing along with urbanisation, rising incomes and the ageing of the population. ${ }^{15}$ China has made substantial improvements in hypertension treatment indicators such as blood pressure lowering medication use and blood pressure control over the past decades. ${ }^{16}{ }^{17}$ However, despite these improvements nearly half of the elderly hypertensive participants in our study were not undergoing treatment with antihypertensive medication. In addition, nearly seven in ten of hypertensives who had previously been diagnosed had blood pressure that was uncontrolled.

Several previous epidemiological studies have reported on the treatment of hypertension in Chinese elderly populations. ${ }^{18}{ }^{19}$ One of those studies, the China Health and Retirement Longitudinal Study, measured the treatment of hypertension in a nationally representative sample of 9357 Chinese aged 45 years or above, which provided the best comparison data for our study. ${ }^{18}$ When compared with the findings from that study, the treatment of hypertension in this study indicated higher incidence, $51.0 \%$ vs $54.4 \%$, respectively. ${ }^{18}$ Other regional studies have also previously examined the treatment of hypertension in local elderly residents. Cao $e t a l^{19}$ revealed that the rate of treatment of hypertension in Hebei province was $38.2 \%$, while Du et $a l^{20}$ found that rate in Zhejiang province was $45.4 \%$, both lower than in our study in Shenzhen. This could be because Shenzhen has established a people-centred integrated care model comprising local community health centres. ${ }^{21}$ Under the strong leadership of the district government, comprehensive strategies have been adopted to strengthen primary care and care coordination, improve the quality and efficiency of healthcare delivery, and promote population health. ${ }^{21}$
Even so, the treatment rate of hypertension in the elderly population in Shenzhen was still far below that of elderly in other countries such as the USA, Australia, Germany and Colombia. ${ }^{22-24}$

Similar to other studies, we found that the treatment of hypertension was significantly higher among older, ex-smokers, non-drinkers, higher education level and those engaging in physical activity. ${ }^{9} 192425$ Participants engaged in physical activity may generally pay more attention to their health. Unsurprisingly, therefore, the group with the highest level of physical activity had a higher likelihood of being aware of their hypertension and receiving medication.

Our study revealed a higher level of treatment in individuals with comorbidities such as overweight, obesity, central obesity, diabetes and dyslipidaemia, which are common comorbidities with hypertension. ${ }^{1923}$ Moreover, in our study, history of CVD was highly correlated with receiving antihypertensive treatment. Other researchers have confirmed that having another medical condition, for example, diabetes or dyslipidaemia, is associated with greater awareness and treatment of hypertension. ${ }^{2627}$ This causes people to go to health centres, and consequently be diagnosed with hypertension sooner, obtaining earlier treatment. Therefore, often patients with comorbid diseases have higher perception of the risk factors and their condition. An alternate theory to explain the higher rates of no treatment in individuals with fewer comorbidities is that physicians may be wary of the consequences of initiating medication and quality of life by 'medicalising' an otherwise healthy person. ${ }^{22}$

Compared with previous epidemiological data of hypertension in China, the control rate in our study was significantly higher than the control rate of hypertension among elderly in Hebei province investigated in $2015 .^{19}$ This could be due to the fact that Shenzhen is a relatively well-developed economy with higher levels of education, better community medical facilities and 
Table 3 Risk factor analysis on the treatment and control of hypertension in older adults living in Shenzhen

\begin{tabular}{|c|c|c|c|c|}
\hline \multirow[b]{2}{*}{ Characteristics } & \multicolumn{2}{|c|}{ Treatment $^{*}$} & \multicolumn{2}{|c|}{ Control† } \\
\hline & OR $(95 \% \mathrm{Cl})$ & $P$ value & OR $(95 \% \mathrm{Cl})$ & $P$ value \\
\hline \multicolumn{5}{|l|}{ Sex } \\
\hline Male & - & - & 1.00 (Reference) & \\
\hline Female & - & - & 0.91 (0.88 to 0.95$)$ & $<0.01$ \\
\hline \multicolumn{5}{|l|}{ Education level } \\
\hline Illiterate & 1.00 (Reference) & & 1.00 (Reference) & \\
\hline Primary education & $1.00(0.95$ to 1.07$)$ & 0.90 & 0.99 (0.93 to 1.06$)$ & 0.84 \\
\hline Junior school education and above & 1.25 (1.18 to 1.32$)$ & $<0.01$ & 1.28 (1.20 to 1.36$)$ & $<0.01$ \\
\hline \multicolumn{5}{|l|}{ Marital status } \\
\hline Married or cohabiting & 1.00 (Reference) & & - & - \\
\hline Widowed & $1.28(1.17$ to 1.40$)$ & $<0.01$ & - & - \\
\hline Divorced & 1.07 (0.87 to 1.31$)$ & 0.54 & - & - \\
\hline Single & 0.58 (0.45 to 0.74$)$ & $<0.01$ & - & - \\
\hline \multicolumn{5}{|l|}{ Age group } \\
\hline $65 \sim$ & 1.00 (Reference) & & 1.00 (Reference) & \\
\hline $70 \sim$ & $1.11(1.07$ to 1.15$)$ & $<0.01$ & 0.98 (0.94 to 1.02$)$ & 0.23 \\
\hline $75 \sim$ & 1.24 (1.18 to 1.30$)$ & $<0.01$ & 0.96 (0.92 to 1.01$)$ & 0.13 \\
\hline $80 \sim$ & 1.32 (1.25 to 1.38$)$ & $<0.01$ & 0.93 (0.88 to 0.98$)$ & $<0.01$ \\
\hline \multicolumn{5}{|l|}{ Physical activity } \\
\hline No & 1.00 (Reference) & & 1.00 (Reference) & \\
\hline Yes & $1.14(1.10$ to 1.18$)$ & $<0.01$ & 1.06 (1.02 to 1.10$)$ & $<0.01$ \\
\hline \multicolumn{5}{|l|}{ Smoking status } \\
\hline Never-smoker & 1.00 (Reference) & & - & - \\
\hline Current smoker & 1.06 (1.00 to 1.13$)$ & 0.06 & - & - \\
\hline Ex-smoker & 1.19 (1.11 to 1.27$)$ & $<0.01$ & - & - \\
\hline \multicolumn{5}{|l|}{ Drinking habit } \\
\hline Non-drinker & 1.00 (Reference) & & 1.00 (Reference) & \\
\hline Non-habitual drinker & 0.89 (0.85 to 0.94$)$ & $<0.01$ & 0.92 (0.87 to 0.97$)$ & $<0.01$ \\
\hline Habitual drinker & 0.72 (0.68 to 0.77$)$ & $<0.01$ & 0.73 (0.68 to 0.79$)$ & $<0.01$ \\
\hline \multicolumn{5}{|l|}{ History of cardiovascular disease } \\
\hline No & 1.00 (Reference) & & 1.00 (Reference) & \\
\hline Yes & 2.20 (2.04 to 2.37$)$ & $<0.01$ & 1.82 (1.71 to 1.96$)$ & $<0.01$ \\
\hline \multicolumn{5}{|l|}{ BMI } \\
\hline Low weight & 1.00 (Reference) & & - & - \\
\hline Normal weight & $1.42(1.28$ to 1.57$)$ & $<0.01$ & - & - \\
\hline Overweight & 1.64 (1.48 to 1.82$)$ & $<0.01$ & - & - \\
\hline Obesity & 1.89 (1.68 to 2.11$)$ & $<0.01$ & - & - \\
\hline \multicolumn{5}{|l|}{ Central obesity } \\
\hline No & 1.00 (Reference) & & 1.00 (Reference) & \\
\hline Yes & 1.10 (1.06 to 1.14$)$ & $<0.01$ & 0.94 (0.91 to 0.98$)$ & $<0.01$ \\
\hline \multicolumn{5}{|l|}{ Diabetes } \\
\hline No & 1.00 (Reference) & & 1.00 (Reference) & \\
\hline Yes & 1.49 (1.44 to 1.54$)$ & $<0.01$ & 1.52 (1.47 to 1.58$)$ & $<0.01$ \\
\hline \multicolumn{5}{|l|}{ Dyslipidaemia } \\
\hline No & 1.00 (Reference) & & 1.00 (Reference) & \\
\hline
\end{tabular}


Table 3 Continued

\begin{tabular}{|c|c|c|c|c|}
\hline \multirow[b]{2}{*}{ Characteristics } & \multicolumn{2}{|c|}{ Treatment* } & \multicolumn{2}{|c|}{ Controlt } \\
\hline & OR (95\% Cl) & $P$ value & OR (95\% Cl) & $P$ value \\
\hline Yes & $1.20(1.16$ to 1.24$)$ & $<0.01$ & 1.05 (1.03 to 1.09$)$ & $<0.01$ \\
\hline
\end{tabular}

Definition: A diagnosis of hypertension was considered when three consecutive high readings ( $\geq 140$ systolic and/or $\geq 90$ $\mathrm{mm} \mathrm{Hg}$ diastolic) with 2-week intervals were registered, or treatment with antihypertensive medication within the previous 2 weeks was self-reported. Participants were considered to be treated if they answered 'yes' to the question 'Because of your high blood pressure/hypertension, are you now taking prescribed medicine?' Controlled hypertension was defined as SBP $<140 \mathrm{~mm} \mathrm{Hg}$ and DBP $<90 \mathrm{~mm} \mathrm{Hg}$, and reported use of antihypertensive medication during the survey.

${ }^{*}$ Adjusted for sex.

†Adjusted for marital status, smoking status and BMI.

BMI, body mass index; DBP, diastolic blood pressure; SBP, systolic blood pressure.

equipment, and relatively high levels of diagnosis and treatment for hypertension compared with the national average. However, the control rate of hypertension in Shenzhen elderly were much lower than those reported in developed countries. ${ }^{22}{ }^{23}$ Possible reasons include the following: (1) Shenzhen medical institutions are not doing a good job of screening for hypertension; ${ }^{28}$ (2) Hypertensive individuals often cease taking antihypertensive agents when blood pressure control has been achieved, which may result in uncontrolled hypertension when checked later unless there is regular monitoring; ${ }^{29}$ (3) Primary care physicians in the China might have been less knowledgeable or experienced in diagnosing and treating hypertension compared with those in developed countries; ${ }^{29}$ (4) China's doctors might have become entrenched in traditional prescription practice and lack knowledge or willingness to follow new guidelines due to obstacles in information exchange and ${ }^{29}$ (5) There was a lower rate of out-of-office blood pressure monitoring among hypertensive patients in China than that in developed countries, in spite of recommendations in multiple guidelines. ${ }^{30} 31$

Previous studies have revealed that being female had a statistically significant association with the control of hypertension. ${ }^{24}$ On the contrary, our findings suggested that being female was a negative indicator of hypertension control, strongly indicating that it deserves further study. Our study revealed that participants with high educational attainment had more than 1.28-fold higher probability of being in control of their hypertension status than those with low educational level. Education is a well-elucidated determinant of health disparity, and such disparities have been shown to be more pronounced in later life phases. ${ }^{32}$ In the present study, hypertension control decreased with age. Literature shows consistency in the association between age and control of hypertension. ${ }^{33}$ Older people often have hypertension accompanied by multiple diseases, as well as cognitive decline and low medication compliance, which are all related to the lower control rate of hypertension. ${ }^{34}$ Previous studies have found that older age was independently associated with greater hypertension awareness and treatment but poorer hypertension control, which is in accordance with the current report. ${ }^{35} 36$

Alcohol consumption and physical inactivity have also been correlated with inferior hypertension control. As Gooding et $a l^{37}$ reported that patients with more unhealthy behaviours care less about subjective wellbeing. This may have led to a generally lower control rate. In addition, the control of hypertension was lower with higher WC in our study, consistent with some previous studies. ${ }^{19} 38$ Greater WC is correlated with higher levels of fat mass, an increase in salt retention and insulin resistance, which cause increased high blood pressure. ${ }^{39}$

In our study, patients who had diabetes or dyslipidaemia had higher probability of having controlled blood pressure, consistent with other studies. ${ }^{25}{ }^{40} \mathrm{An}$ explanation might be that when people have diabetes or dyslipidaemia, they become more focused on their health, and therefore may be more likely to engage in and comply with blood pressure-lowering drugs or lifestyle intervention for hypertension. Our study additionally showed that history of CVD is a positive factor for effective blood pressure control. Physicians use more ACE inhibitors, angiotensin receptor blockers or even aldosterone receptor blockers to treat participants with CVD, which all contribute to effective blood pressure reduction. $^{41}$

Our study had some limitations. First, given the cross-sectional nature of the study design, only associations, rather than causality, could be inferred. Second, our research enrolled the elderly population by convenience sampling. This is a major factor preventing true extrapolation of the results to the general population. Third, patient previous experience of medications for other conditions could have contributed to their current adherence to treatment. Further studies are needed to evaluate the correlation between patient previous experience of taking medication and current hypertension treatment. Fourth, the impact of white coat hypertension and masked hypertension could not be excluded as factors affecting the findings in our research. 


\section{CONCLUSION}

In conclusion, we found a high prevalence of hypertension but a low prevalence of treatment and control among Shenzhen elderly, a group at high risk for future CVD events. This study represents a warning for cardiovascular health management in Shenzhen elderly. Improvement in hypertension treatment and control should be a public health priority to reduce the disproportionate burden of CVD in this growing population. Out-of-office blood pressure monitoring is one measure that could be introduced to identify hypertensive patients earlier so as to start treatment more promptly to reduce CVD incidence in this high-risk group.

Acknowledgements We are grateful to all the volunteers for participating in the present study, and to all the investigators for their support and hard work during this survey.

Contributors WN and JX: study conception and design. WN, XY, JZ, PL, HMZ, YZ and JX: performance of research. $X Y$ and JZ: data analysis and interpretation. WN: writing the original draft. WN and JX: writing the review and editing. All authors have read and agreed to the published version of the manuscript.

Funding This study was supported by the Science and Technology Planning Project of Shenzhen City, Guangdong Province, China (Grant No. SZGW2018002); the Science and Technology Planning Project of Shenzhen City, Guangdong Province, China (Grant No. JCYJ20180703145202065); and the Shenzhen Medical Key Discipline Construction Fund, and the Sanming Project of Medicine in Shenzhen (Grant No. SZSM201811093).

\section{Competing interests None declared.}

Patient and public involvement Patients and/or the public were not involved in the design, or conduct, or reporting, or dissemination plans of this research.

\section{Patient consent for publication Not required.}

Ethics approval The study received ethnicity approval from the Center for Chronic Disease Control in Shenzhen(Grant No: SZCCC-201802, SZCCC-2020-018-01-PJ).

Provenance and peer review Not commissioned; externally peer reviewed.

Data availability statement № data are available. № additional data are available.

Open access This is an open access article distributed in accordance with the Creative Commons Attribution Non Commercial (CC BY-NC 4.0) license, which permits others to distribute, remix, adapt, build upon this work non-commercially, and license their derivative works on different terms, provided the original work is properly cited, appropriate credit is given, any changes made indicated, and the use is non-commercial. See: http://creativecommons.org/licenses/by-nc/4.0/.

\section{ORCID iD}

Jian Xu http://orcid.org/0000-0001-5727-8191

\section{REFERENCES}

1 James PA, Oparil S, Carter BL, et al. 2014 evidence-based guideline for the management of high blood pressure in adults: report from the panel members appointed to the eighth joint National Committee (JNC 8). JAMA 2014;311:507-20.

2 Lloyd-Jones DM, Evans JC, Levy D. Hypertension in adults across the age spectrum: current outcomes and control in the community. JAMA 2005;294:466-72.

3 Franklin SS, Larson MG, Khan SA, et al. Does the relation of blood pressure to coronary heart disease risk change with aging? The Framingham heart study. Circulation 2001;103:1245-9.

4 Wang HM. [Attaching importance to health of elderly population and promoting national healthy ageing actively in China]. Zhonghua Liu Xing Bing Xue Za Zhi 2019;40:259-65.

5 Oliveros E, Patel H, Kyung S, et al. Hypertension in older adults: assessment, management, and challenges. Clin Cardiol 2020;43:99-107.

6 Kantomaa MT, Stamatakis E, Kankaanpää A, et al. Physical activity and obesity mediate the association between childhood motor function and adolescents' academic achievement. Proc Natl Acad Sci U S A 2013;110:1917-22.

7 Zhang L, Wang F, Wang L, et al. Prevalence of chronic kidney disease in China: a cross-sectional survey. Lancet 2012;379:815-22.

8 Zhang M, Liu S, Yang L. Prevalence of smoking and knowledge about the smoking hazards among 170,000 Chinese adults: a nationally representative survey in 2013-2014. Nicotine Tob Res 2019;21:1644-51.

9 Writing Group of 2018 Chinese Guidelines for the Management of Hypertension, Chinese Hypertension League, Chinese Society of Cardiology, Chinese Medical Doctor Association Hypertension Committee, Hypertension Branch of China International Exchange and Promotive Association for Medical and Health Care. 2018 Chinese guidelines for the management of hypertension. Chin J Cardiovasc Med 2019;24:24-56.

10 Qaseem A, Wilt TJ, Rich R, et al. Pharmacologic treatment of hypertension in adults aged 60 years or older to higher versus lower blood pressure targets: a clinical practice guideline from the American College of physicians and the American Academy of family physicians. Ann Intern Med 2017;166:430-7.

11 Wang Q, Zhang X, Fang L, et al. Prevalence, awareness, treatment and control of diabetes mellitus among middle-aged and elderly people in a rural Chinese population: a cross-sectional study. PLOS One 2018;13:e0198343.

12 Joint committee for guideline revision. 2016 Chinese guidelines for the management of dyslipidemia in adults. $J$ Geriatr Cardiol 2018;15:1-29.

13 GBD 2013 Mortality and Causes of Death Collaborators. Global, regional, and national age-sex specific all-cause and causespecific mortality for 240 causes of death, 1990-2013: a systematic analysis for the global burden of disease study 2013. Lancet 2015;385:117-71.

14 GBD 2016 Causes of Death Collaborators. Global, regional, and national age-sex specific mortality for 264 causes of death, 19802016: a systematic analysis for the global burden of disease study 2016. Lancet 2017;390:1151-210.

15 Yang G, Wang Y, Zeng Y, et al. Rapid health transition in China, 19902010: findings from the global burden of disease study 2010. Lancet 2013;381:1987-2015.

16 Wu Y, Huxley R, Li L, et al. Prevalence, awareness, treatment, and control of hypertension in China: data from the China national nutrition and health survey 2002. Circulation 2008;118:2679-86.

17 Wang Z, Chen Z, Zhang L, et al. Status of hypertension in China: results from the China hypertension survey, 2012-2015. Circulation 2018;137:2344-56.

18 Li C, Lumey LH. Impact of disease screening on awareness and management of hypertension and diabetes between 2011 and 2015: results from the China health and retirement longitudinal study. BMC Public Health 2019;19:421.

19 Cao YJ, Qi SF, Yin HS, et al. [Prevalence, awareness, treatment and control of hypertension in elderly residents in Hebei province]. Zhonghua Liu Xing Bing Xue Za Zhi 2019;40:296-300.

20 Du XF, Chen XY, Zhang J, et al. [Prevalence, control of hypertension and intake of sodium and potassium among residents aged 50-69 years old in Zhejiang Province in 2017]. Zhonghua Yu Fang Yi Xue Za Zhi 2019;53:464-9.

21 Liang D, Mei L, Chen Y, et al. Building a people-centred integrated care model in urban China: a qualitative study of the health reform in Luohu. Int J Integr Care 2020;20:9.

22 Chowdhury EK, Nelson MR, Ernst ME, et al. Factors associated with treatment and control of hypertension in a healthy elderly population free of cardiovascular disease: a cross-sectional study. Am J Hypertens 2020;33:350-61.

23 Muli S, Meisinger C, Heier M, et al. Prevalence, awareness, treatment, and control of hypertension in older people: results from the population-based KORA-age 1 study. BMC Public Health 2020;20:1049.

24 Barrera L, Gómez F, Ortega-Lenis D, et al. Prevalence, awareness, treatment and control of high blood pressure in the elderly according to the ethnic group. Colombian survey. Colomb Med 2019;50:115-27.

25 Rajati F, Hamzeh B, Pasdar Y, et al. Prevalence, awareness, treatment, and control of hypertension and their determinants: results from the first cohort of non-communicable diseases in a Kurdish settlement. Sci Rep 2019;9:12409.

26 Malekzadeh MM, Etemadi A, Kamangar F, et al. Prevalence, awareness and risk factors of hypertension in a large cohort of Iranian adult population. $J$ Hypertens 2013;31:1364-71.

27 Ware LJ, Chidumwa G, Charlton K, et al. Predictors of hypertension awareness, treatment and control in South Africa: results from 
the WHO-SAGE population survey (wave 2). $J$ Hum Hypertens 2019;33:157-66.

28 LV D, Feng T, Yuan X. The current status of information platform of office blood pressure monitoring for first-time visits in the public general hospitals in Shenzhen. Chin J Hypertens 2017;25:554-8.

29 Wang Z, Wang X, Chen Z, et al. Hypertension control in community health centers across China: analysis of antihypertensive drug treatment patterns. Am J Hypertens 2014;27:252-9.

30 Zhu H, Liang X, Pan X-F, et al. A prospective cohort study of home blood pressure monitoring based on an intelligent cloud platform (the HBPM-iCloud study): rationale and design. Ther Adv Chronic Dis 2020;11:2040622320933108.

31 Chinese Working Group on Blood pressure Measurement. Chinese guidelines for nlood pressure measurement. Chin J Hypertens 2011:19:1101-15.

32 Oshio T. Widening disparities in health between educational levels and their determinants in later life: evidence from a nine-year cohort study. BMC Public Health 2018;18:278.

$33 \mathrm{Wu} \mathrm{L}, \mathrm{He} \mathrm{Y}$, Jiang B, et al. Trends in prevalence, awareness, treatment and control of hypertension during 2001-2010 in an urban elderly population of China. PLoS One 2015;10:e0132814.

34 Chinese Society of Geriatrics Hypertension Branch. China experts consensus on the managements of hypertension in the very old people. Chin J Cardiovasc Med 2015;20:401-9.

35 Wang $\mathrm{H}$, Zhang $\mathrm{X}$, Zhang J, et al. Factors associated with prevalence, awareness, treatment and control of hypertension among adults in southern China: a community-based, cross-sectional survey. PLoS One 2013;8:e62469.

36 Muntner P, Gu D, Wu X, et al. Factors associated with hypertension awareness, treatment, and control in a representative sample of the Chinese population. Hypertension 2004;43:578-85.

37 Gooding HC, McGinty S, Richmond TK, et al. Hypertension awareness and control among young adults in the National longitudinal study of adolescent health. J Gen Intern Med 2014;29:1098-104.

38 Tapela NM, Clifton L, Tshisimogo G, et al. Prevalence and determinants of hypertension awareness, treatment, and control in Botswana: a nationally representative population-based survey. Int $J$ Hypertens 2020;2020:1-12.

39 Cooper R, Van Horn L, Liu K, et al. A randomized trial on the effect of decreased dietary sodium intake on blood pressure in adolescents. $J$ Hypertens 1984;2:361-6.

40 Lora CM, Ricardo AC, Chen J, et al. Prevalence, awareness, and treatment of hypertension in Hispanics/Latinos with CKD in the Hispanic community health Study/Study of Latinos. Kidney Med 2020;2:332-40.

41 Attar A, Sadeghi A-A, Amirmoezi F, et al. Low dose spironolactone monotherapy in the management of stage I essential hypertension: a pilot randomized, double-blind, placebo-controlled trial. Acta Cardiol Sin 2018;34:59-65. 